\title{
IL LIBRO FITTIZIO: STORIA E FUNZIONI DI UN ESPEDIENTE LETTERARIO
}

\author{
Daniel RAFFINI \\ Università degli Studi di Roma La Sapienza
}

\begin{abstract}
En): The article deals with the topos of fictional books that only exist inside others books. Firstly, a theoretical study about fictional books is presented, such as the ones by Max Beerbohm and by Lyon Sprague de Camp. Then, we focus on the function of fictional books in some authors from the early modern to the contemporary age. The first two trends are the satirical one, from François Rabelais' Pantagruel, and the invention of the sources in Miguel de Cervantes' Don Quijote. During the twentieth century, fictional books acquire more and more functions. We will analyze the cases of Jorge Luis Borges, who uses the fictional book in order to create fantastic worlds, and that of Italo Calvino, who uses books inside books as a metaliterary tool in the novel Se una notte d'inverno un viaggiatore (1979). These and other examples will be analyzed with the aim of understanding the reasons for the great numbers of fictional books over the centuries.
\end{abstract}

Keywords (En): Fictional books ; Functions ; Rabelais ; Cervantes ; Borges ; Calvino

Parole chiave (It): Libri fittizi ; Funzioni ; Rabelais ; Cervantes ; Borges ; Calvino

\section{Introduzione}

Molti libri fittizi popolano le letterature moderne: si tratta di opere citate all'interno di altre opere, libri che in realtà non esistono, scritture di cui viene simulata l'esistenza. All'interno della coppia canonica simulazione-dissimulazione che cataloga i prodotti della menzogna, il libro fittizio si pone sul versante della simulazione, dal momento che finge l'esistenza di qualcosa che non c'è. Si tratta di una menzogna produttiva, che crea nuovo materiale laddove la realtà ne è priva. A ben vedere si può ascrivere al campo della simulazione l'intero universo letterario. Il libro fittizio si presenta in questo senso come una stratificazione di simulazioni: se ogni libro è di per sé una simulazione, inserire un libro fittizio in un libro reale significa simulare l'esistenza di un oggetto simulante all'interno di un altro oggetto simulante. La differenza sta nel fatto che uno di questi oggetti, il libro reale, possiede la qualità dell'esistenza fisica, l'altro, il libro fittizio, ha un'esistenza puramente intellettuale.

Il tema del libro fittizio ha iniziato a interessare studiosi di varia natura a partire dalla metà del XIX secolo. I primi approcci sono quelli di Édouard Fournier e di Pierre Jannet, i cui contributi appaiono tra il 1848 il 1849 sul «Journal de l'amateur de livres». Qualche anno dopo, nel 1851, viene pubblicato il primo studio sistematico sull'argomento, l'Essai sur les bibliothèques imaginaires di Gustave Brunet. Il fenomeno valica presto le Alpi e prima della fine del secolo appaiono in Italia gli studi di Giuseppe Fumagalli: Delle Biblioteche immaginarie $e$ dei libri che non esistono e Biblioteche immaginarie e roghi di libri, in collaborazione con Leo Olschki. In questi primissimi approcci il punto di vista adottato è principalmente quello del curioso e dell'erudito. Studi più organici e 
finalizzati a una definizione più precisa del fenomeno si hanno dall'inizio del Novecento con Max Beerbohm, autore nel 1914 del saggio Books within books e Lyon Spargue De Camp, che nel 1947 pubblica l'articolo The Unwritten Classics. Vale la pena soffermarsi brevemente su questi due contributi, che pongono le basi per uno studio più scientifico del fenomeno.

Beerbohm definisce i libri fittizi con il termine greco abiblia e inizia la sua trattazione parlando dell'effetto sul lettore. I libri fittizi, nella loro incompletezza e frammentarietà, nel loro essere racconto di un racconto ipotetico, destano nei lettori una curiosità tale da determinare che «in nine cases out of ten, a book within a book is an immediate, an immense success» (BEERBOHM, 1914). Beerbohm si sofferma poi sullo scrittore e afferma che in lui avrebbe luogo una sorta di transfert nei confronti del personaggio, su cui proietterebbe il proprio desiderio di scrivere un libro di successo. Tale spiegazione, più sul versante della leggerezza ironica che su quello della psicanalisi, non prescinde però da un'analisi empirica del fenomeno. I libri fittizi - dice Beerbohm basandosi su un canone prettamente inglese - sono il più delle volte raccolte di poesie che gli autori attribuiscono ai loro personaggi. Questo tipo di libri fittizi si lega strettamente al tipo di personaggi presentati: giovani poeti squattrinati alla ricerca di un improbabile successo. Tale condizione permette l'immedesimazione e la partecipazione del lettore: «Their spoken thoughts have seemed to me no more profound or pungent than my own» (BEERBOHM, 1914). Insomma Beerbohm inizia, seppur in maniera del tutto personale e indiretta, a delineare una delle funzioni del libro fittizio - procedimento centrale per lo studio di questo espediente letterario. Nella parte restante del saggio Beerbohm si sofferma su vari romanzi nei quali appaiono libri fittizi, fornendoci una buona istantanea del fenomeno per quanto riguarda la letteratura inglese tra la fine dell'Ottocento e gli inizi del Novecento.

Ben più ampia è la prospettiva adottata da Sprague de Camp. Egli sente prima di tutto il bisogno di classificare i libri irreali, che possono rientrare in differenti categorie: «Between the wholly real and the wholly unreal books lies a shadowland of books that are, yet are not: unfinished books, lost books, apocrypha, and pseudepigrapha (falsely attributed books)» (SPRAGUE DE CAMP, 1947: 7). Tale volontà classificatoria diventerà uno dei punti più importanti nei successivi studi teorici sul fenomeno, con lo scopo di arrivare a una definizione precisa di libro fittizio. Dopo aver introdotto varie tipologie, Sprague de Camp restringe il campo e definisce gli pseudobiblia o unwritten classics come «books that were never written, but which exist solely as a title, with perhaps excerpts, in a work of fiction or pseudo-fact» (Ibidem). Subito dopo precisa, come aveva già fatto Beerbohm che «some of these last have led an active literary career despite the fact that they never existed» (Ibidem). Una volta definito l'oggetto di studio, l'autore delinea le origini del fenomeno: se Beerbohm si limitava alla letteratura inglese contemporanea, Sprague de Camp risale fino all'antico Egitto. Il fenomeno appare dunque radicato nelle origini stesse della scrittura e della letteratura. Il primo pseudobibion sarebbe stato il Libro di Thot, che appare nel racconto egiziano La storia di Setnau Khaemuast. Partendo dal Libro di Thot Sprague de Camp si ricollega al Necronomicon di Lovecraft, il libro di magia nera che appare in molti 
dei racconti fantastici dello scrittore statunitense. Sprague de Camp continua poi sulla linea dei libri fittizi della letteratura fantastica, citando il racconto Brood of the Witch Queen di Sax Rohmer, Merlin and Vivien di Tennyson e il fittizio Book of Dzyan di M.me Blavatsky. Sprague de Camp individua una continuità tra la contemporanea letteratura fantastica e il libro attribuito al dio egizio Thot, che in seguito sarebbe passato in Grecia sotto forma di Hermes per poi approdare al medioevo arabo e cristiano come pseudo-autore dei famosi testi ermetici. In ognuna di queste metamorfosi Thot resta l'autore di libri fittizi di magia e di alchimia, libri che proprio grazie a questa lunga tradizione hanno potuto, nella visione di Sprague de Camp, influenzare la contemporanea letteratura fantastica. ${ }^{1}$ Anche se la lettura risulta azzardata, il contributo rimane importante perché per la prima volta viene considerata la continuità del libro fittizio dall' antichità fino alla contemporaneità.

In seguito all'intervento di Sprague de Camp la questione libro fittizio trova sempre più appassionati tra gli studiosi di letteratura e appaiono molti saggi, tra cui ricordiamo Imaginary books and library di John Webster Spargo (1952) e Imaginary books and phantom libraries di Walter Har Blumenthal (1966). A interessarsi al fenomeno sono anche gli studiosi di bibliografia. Contributi sui libri e sulle biblioteche immaginarie appaiono ad esempio nella Storia della bibliografia edita nel 1993 da Bulzoni, nell' «Almanacco del bibliofilo» e nel Manuale Enciclopedico della Bibliofilia (1997), curato da Vittorio Di Giuro. Negli ultimi decenni gli studi hanno fatto un ulteriore passo avanti. Dopo la fase teorica si è passati all'elaborazione di strumenti di consultazione, come i cataloghi di libri fittizi. Essi sono sia in forma digitale, come la «Invisibile Library», buona fonte soprattutto per l'area anglosassone, che in forma cartacea, come il volume Mirabilia. Catalogo ragionato di libri introvabili curato da Paolo Albani e Paolo Della Bella.

A fronte di tante classificazioni e definizioni, sarà utile dire ciò che intenderemo qui per libro fittizio. La definizione che seguiremo è quella di Sprague de Camp, che considerava i libri fittizi propriamente detti come quei libri immaginari che vengono citati o descritti all'interno di libri reali. La caratteristica principale dei libri fittizi è dunque quella di non esistere come libri reali ma solo come espedienti letterari. La nozione di espediente ben si confà al libro fittizio perché rimanda all'utilizzo di un artificio che abbia un determinato ruolo all'interno dell'azione narrativa. In qualità di espedienti letterari, i libri fittizi ricoprono infatti delle funzioni all'interno dei libri reali che li contengono; l'individuazione di tali funzioni è ciò che manca negli studi sul libro fittizio. Alcuni critici e commentatori vi hanno accennato, tuttavia non esiste ancora uno studio sistematico che metta in rapporto l'evoluzione storica di questo espediente

\footnotetext{
${ }^{1}$ Oltre alla letteratura fantastica, l'espediente del libro fittizio è rilevante anche nella letteratura fantasy. Qui il libro fittizio è un elemento centrale e si affianca agli altri elementi che vanno a comporre il mondo fantasy di un determinato autore o di una certa saga. Nel momento di creare un mondo, gli autori di fantasy devono creare anche i libri di quel mondo. Nascono così la mitologia della Terra di Mezzo di Tolkien, la biblioteca di Tumnus de Le Cronache di Narnia, l'Enciclopedia Galattica di Asimov o i libri magici citati nella saga di Harry Potter.
} 
letterario con le funzioni che di volta in volta ha assunto. Proveremo allora a ricostruirne qui alcune ponendoci sull'asse cronologico delle letterature moderne.

\section{Rabelais e la funzione satirica}

Per quanto riguarda la modernità letteraria, la prima funzione che assume l'espediente del libro fittizio è quella satirica. Questa resterà preponderante almeno fino alla metà dell'Ottocento, come testimonia Brunet nel suo Essai sur les bibliothèques imaginaries del 1851. Seppur datato, il saggio di Brunet appare utile come documento storico, perché ci presenta la percezione del fenomeno alla metà dell'Ottocento, prima delle grandi innovazioni di cui l'espediente del libro fittizio sarà protagonista nel corso del XX secolo. Parlando dei libri fittizi e delle biblioteche immaginarie, Brunet dice che i loro ideatori li avrebbero inventati «obéissant presque toujours à des intentions satirique» (BRUNET, 1851: 1). I libri fittizi non sarebbero dunque da prendere sul serio, dal momento che il loro carattere ironico appare evidente. Brunel attribuisce «l'invention de ce genre de sarcasmes» (BRUNET, 1851: 1) a François Rabelais. Il riferimento è al catalogo della Biblioteca dell'Abbazia di San Vittore che appare nel VII capitolo del Pantagruel. Questo catalogo immaginario è convenzionalmente considerato il punto d'inizio della storia del libro fittizio in epoca moderna. I libri inventati da Rabelais per la Biblioteca di San Vittore sono sì fittizi, ma allo stesso tempo i titoli - parodistici e ironici - rimandano in maniera più o meno diretta a libri esistenti e rappresentano una caricatura della letteratura erudita dell'epoca. Rabelais in effetti aveva frequentato la vera Biblioteca di San Vittore, così che i titoli da lui inventanti fanno spesso riferimento a libri reali, ripresi attraverso deformazioni grottesche ed evidenti caricature. ${ }^{2}$

Dal punto di vista formale il capitolo sulla Biblioteca di San Vittore si caratterizza per l'utilizzo dell'elencazione. I libri sono presentati solamente attraverso i titoli, senza spiegazioni sui loro contenuti. Tuttavia gli argomenti di questi libri fittizi appaiono evidenti dai titoli ed evidente è anche il fine parodico e umoristico. I titoli sono in realtà delle caricature di titoli e l'intera biblioteca, nella sua assurdità, si configura come una satira del collezionismo e della bibliofilia. Dunque Rabelais è importante all'interno della storia del libro fittizio sia per l'utilizzo dell'elencazione - da cui nasce la feconda tradizione dei cataloghi di biblioteche immaginarie - sia per l'altrettanto fortunata funzione parodica.

A conferma della lunga fortuna della funzione parodica testimoniata da Brunet c'è tutta la tradizione ludico-satirica che inizia nel XVI secolo e che va avanti almeno fino al XIX. In questi secoli si moltiplicano gli esempi di biblioteche fittizie, che rientrano nel gusto per i gabinetti delle meraviglie e per lo stravagante, a cui si uniscono spesso le burle degli scrittori e degli editori. ${ }^{3}$ I cataloghi puri quelli non inseriti in altri libri - perdono però il carattere di espediente letterario e

\footnotetext{
${ }^{2}$ Cfr. Lacroix Paul (1862), Catalogue de la Bibliothèque de Saint-Victor au sezième siècle rédigé par François Rabelais, Paris, J. Techener.

3 Tra le burle ai danni di bibliofili e collezionisti si ricorda il catalogo dei libri della biblioteca del Conte di Fortsas (1840) e quello della biblioteca del signor Edmond Cuénoud (1910).
} 
non hanno più quella funzione all'interno di un'azione narrativa che invece era presente in Rabelais. Nonostante ciò, anche i cataloghi immaginari riprendono la funzione parodica e satirica di ascendenza rabelesiana. Tra i primi a pubblicare in forma autonoma un catalogo di libri immaginari c'è il poeta tedesco Johann Fischart con il suo Catalogus catalogorum perpetuo durabilis, pubblicato nel 1590, cui segue il Catalogus librorum auliorum incomparabilium et not vendibilium (1603-11) di John Donne. Anche solo limitandosi ai titoli di tali cataloghi, appare evidente il loro carattere ironico.

Altro caso interessante di funzione satirica in questi secoli è quello dei libri fittizi che appaiono in Tristram Shandy di Laurence Sterne, sia attributi al protagonista che citati come sue letture. A differenza di Rabelais, Sterne non si avvale di un catalogo, abbandona la forma dell'elencazione e inserisce i libri fittizi all'interno della narrazione. Il fine è però ancora quello rabelesiano di parodiare l'erudizione del tempo, a cui si aggiunge la volontà di caratterizzare il personaggio di Tristram attraverso le sue fantasiose letture e i suoi ipotetici lavori letterari. Tra i secoli XVI e XIX sono poi molti altri gli esempi di funzione satirica, che qui non si potranno approfondire e che citiamo solo di passaggio: tra di essi si ricorderanno almeno La seconda libraria (1551) di Anton Francesco Doni, La biblioteca faceta e chiribizzosa (1588) di Vincenzo Belando, la Bibliotheca ascondita (1683) di Thomas Browne, la Libreria de' Gastrimargi (1689) di Francesco Fulvio Frugoni e l'interessante caso de Le curiose recensioni pubblicate da Giovan Pellegrino Dandi sul «Gran Giornale de’ Letterati» tra il 1701 e il 1705.

La funzione satirica rimane attiva anche durante il XX secolo, quando il libro fittizio assumerà anche nuove funzioni. Ne è un esempio il catalogo dei Cens bons libres di journaliste che appare nel libro Moeurs de Diurnales. Traité de journalisme di Marcel Schwob (1903), per il quale lo stesso autore ammette il modello rabelesiano e il carattere ironico. Dunque il libro fittizio nasce e si sviluppa nel contesto della satira e mantiene a lungo un carattere prettamente ironico e ludico. La finzione può essere apertamente manifesta, come nel caso dei cataloghi di titoli improbabili, oppure fingersi verità, soprattutto quando i cataloghi sono fatti a scopo di scherzo; in ogni caso, non mancano mai delle spie, elementi anche minimi, a fungere da indici di finzionalità.

\section{Cervantes: l'invenzione delle fonti e il manoscritto ritrovato}

Sulla funzione parodica si fonda anche un altro testo capitale della letteratura moderna, il Don Quijote di Miguel de Cervantes, libro-parodia dei libri di cavalleria di moda all'epoca. Il Quijote è però un libro reale, così come sono reali i libri presenti nella biblioteca del protagonista descritti nel capitolo VI della prima parte del romanzo. Anche qui la biblioteca si lega alla funzione satirica: i libri della biblioteca di Don Quijote altro non sono se non i libri parodiati da Cervantes per mezzo del suo romanzo.

All'interno del Don Quijote troviamo anche dei libri fittizi veri e propri, la cui funzione è diversa da quella satirica e che danno vita ad un altro filone, parallelo a quello rabelesiano e non meno importante. Si tratta dell'espediente del manoscritto ritrovato, altra possibile declinazione del libro fittizio. La dialettica tra realtà e 
finzione, originale e falso, appare manifesta nel romanzo di Cervantes fin dal prologo, in cui l'autore inscena un dialogo con un interlocutore fittizio, il quale, vedendolo in difficoltà, gli propone di falsificare i sonetti di apertura, attribuendoli a scrittori noti:

Lo primero en que reparáis de los sonetos, epigramas o elogios que os faltan para el principio, y que sean de personajes graves y de título, se puede remediar en que vos mismo toméis algún trabajo de hacerlos, y después los podéis bautizar y ponerle el nombre que quisiéredes, ahijándolos al Preste Juan de las Indias o al emperador de Trapisonda, de quien yo sé que hay noticia que fueron famosos poetas. (CERVANTES, 2005, p.11)

Nella prosecuzione di questo immaginario dialogo, l'interlocutore consiglia all'autore di compilare una bibliografia di fonti false, non perché fittizie ma perché in realtà non usate dall'autore. Lo scopo dichiarato di tale finzione sarebbe quello di «dar de improviso autoridad al libro» (CERVANTES, 2005: 12). Dunque un tema - quello della simulazione - che appare fin dalle prime pagine del romanzo e che tornerà sotto forma di libro fittizio nel finale del capitolo VIII, quando la narrazione improvvisamente si interrompe nel bel mezzo di un combattimento e l'autore - o, come si definisce, il "secondo autore" - commenta:

Pero está el daño de todo esto que en este punto y término deja pendiente el autor desta historia esta batalla, disculpándose que no alló más escritos destas hazañas de don Quijote, de las que deja referidas. Bien es verdad que el segundo autor desta obra no quiso creer que tan curiosa historia estuviese entregada a las leyes del olvido, ni que hubiesen sido tan poco curiosos los ingenios de la Mancha, que no tuviesen en sus archivos o en sus escritos algunos papeles que deste famoso caballero tratasen; y así, con esta imaginación, no se desesperó de hallar el fin desta apacible historia. (CERVANTES, 2005: 50)

Veniamo dunque a sapere che fino ad allora Cervantes avrebbe seguito una fonte che in questo punto si interrompe. Nelle pagine successive l'autore si mette alla ricerca della continuazione della storia di Don Quijote, che sarà trovata casualmente tra alcune carte in arabo comprate da un ragazzo per strada. La sorpresa nel momento in cui il narratore sente tradurre le carte è incontenibile:

Cuando yo oí decir «Dulcinea del Toboso», quedé atónito y suspenso, porque luego se me representó que aquellos cartapacios contenían la historia de don Quijote. Con esta imaginación le di priesa que leyese el principio, y, haciéndolo ansí, volviendo de improviso el arábigo en castellano, dijo que decía: Historia de don Quijote de la Mancha, escrita por Cide Hamete Benengeli, historiador arábigo. (CERVANTES, 2005: 51)

La storia interrotta può così continuare grazie al ritrovamento provvidenziale del manoscritto. Ci troviamo di fronte al topos del manoscritto ritrovato, che molta fortuna avrà nel corso dei secoli, ma che altro non è se non un libro fittizio che funge da fonte immaginaria per l'opera.

Il Don Quijote è presentato da Cervantes come una stratificazione di fonti che si rivelano fittizie. Seguendo lo spunto dell'interlocutore nel prologo Cervantes inventa le sue fonti, le falsifica. Mancando per deliberato rifiuto le fonti autorevoli, le fonti diventano fittizie, l'autore le inventa. Il fine è doppio: da una parte si vuole dare autorevolezza e veridicità all'opera, dall'altra troviamo di nuovo una finalità 
parodica, che passa attraverso il paradosso di dare veridicità attraverso un testo fittizio. Ancora più ironico se pensiamo che subito dopo Cervantes inserisce una lunga pagina sull'importanza della veridicità nella narrazione storica.

Se in Cervantes il manoscritto ritrovato ha in parte una funzione parodica, nel suo sviluppo questo espediente non può essere equiparato alla linea rabelesiana, giacché tra le due funzioni espresse da Cervantes a prevalere sarà quella dell'autorevolezza, in un'epoca in cui la veridicità si costituisce ancora come valore fondante del lavoro letterario. La testimonianza più scontata di questo ci viene da Manzoni, che usa il manoscritto ritrovato in funzione legittimante. Ma il rapporto stretto tra falsificazione e manoscritto ritrovato sarà tema fertile per gli scrittori del Novecento. Tra i molti possibili, faremo solo l'esempio del romanzo Il consiglio d'Egitto di Leonardo Sciascia, ambientato in una Palermo del Settecento dove vive e agisce un abile falsario, l'abate Giuseppe Vella, che crea dal nulla un antico codice arabo considerato perduto che avrà ripercussioni sulla storia dell'isola. Qui l'espediente del libro fittizio rimanda a una lunga serie di falsi storici, primi fra tutti la donazione di Costantino, che riescono a influire sulla storia nonostante il loro essere delle falsificazioni.

Il libro fittizio si pone dunque fin dalle sue origini su un doppio binario: da una parte la funzione ludica e parodica di matrice rabelesiana, dall'altra quella legittimante, che si esprime attraverso il manoscritto ritrovato. Questa dualità sarà sconvolta nel corso del XX secolo. La rivoluzione culturale e filosofica che investe le arti all'inizio del Novecento determina anche un mutamento di funzioni per il libro fittizio, che dà inizio alla fase di massima fortuna di questo espediente letterario.

\section{I mondi possibili di Jorge Luis Borges}

Con il XX secolo il libro fittizio si apre alla sfera del fantastico e dell'irreale, finendo per esteriorizzare e tematizzare quella che è una delle sue caratteristiche intrinseche. Non solo si simula l'esistenza di un libro, ma il libro fittizio diventa luogo prediletto per fingere l'esistenza di qualcosa che nel mondo reale non esiste o non può esistere, entrando in questo modo nella sfera del fantastico. Uno dei primi e migliori interpreti di questa nuova funzione del libro fittizio è Jorge Luis Borges. Celebre è il suo racconto La Biblioteca di Babele, in cui l'intero universo diventa un'enorme biblioteca. In quel testo Borges afferma che la biblioteca «es total y que sus anaqueles registran todas las posibles combinaciones de los veintitantos símbolos ortográficos» e più avanti continua dicendo che «basta que un libro sea posible para que exista. Sólo está excluido lo imposible» (Borges 2014, p.140). La nozione di possibilità è insomma in Borges abbastanza vasta: possibile è tutto ciò che può essere immaginato, non solo ciò che è reale $\mathrm{o}$ verosimile. Partendo da questo assunto, il libro fittizio diventa per Borges un modo per descrivere i mondi possibili.

Nel prologo del Quijote Cervantes scriveva: «Soy poltrón y perezoso de andarme buscando autores que digan lo que yo me sé decir sin ellos» (CERVANTES, 2005: 10); Borges se vogliamo è ancora più pigro, perché non solo non va a cercare libri che dicano ciò che egli stesso sa dire, ma nemmeno trova 
conveniente descrivere dettagliatamente i mondi possibili che immagina e preferisce fingere che essi siano già stati descritti, in libri che sono per l'appunto fittizi. Questo è ciò che dichiara nel prologo di Ficciones, quando scrive:

Desvarío laborioso y empobrecedor el de componer vastos libros; el de explayar en quinientas páginas una idea cuya perfecta exposicion oral cabe en pocos minutos. Mejor procedimiento es disimular que esos libros ya existen y ofrecer un resumen, un comentario [...]. Más razonable, más inepto, más haragán, he preferido la escritura de notas sobre libros imaginarios. (BORGES, 2014: 89)

Così facendo Borges opera una doppia finzione, inventando libri fittizi che raccontano mondi fittizi. Da qui parte lo scrittore per forgiare un'idea originale di letteratura, che fa un uso del tutto particolare delle fonti. La scrittura borgesiana si fonda su una grande rete bibliografica di testi che in molti casi sono reali e rimandano alle letture dello scrittore, ma che altrettanto spesso sono bibliografie immaginarie, inventate.

La funzione del fantastico è creativa poiché il libro fittizio serve per allargare i confini della realtà, per descrivere tutto ciò che non è ma che potrebbe essere nel presente, nel passato e nel futuro. Tale procedimento appare evidente nel racconto Tlön, Uqbar, Orbis Tertius, in cui Borges racconta una serie di ritrovamenti bibliografici fortuiti che portano alla luce documenti sul paese immaginario di Uqbar. Dalle carte capiamo che i letterati di questa regione avrebbero a loro volta inventato l'universo fittizio di Tlön, descrivendolo nei minimi dettagli in un'enciclopedia di quaranta volumi. Alla fine della riscoperta, l'autore commenta la vastità e l'esaustività di questo mondo fittizio:

Ahora tenía en las manos un vasto fragmento metódico de la historia total de un planeta desconocido, con sus arquitecturas y sus barajas, con el pavor de sus mitologías y el rumor de sus lenguas, con sus emperadores y sus mares, con sus minerales y sus pájaros y sus peces, con su álgebra y su fuego, con su controversia teológica y metafísica. Todo ello articulado, coherente, sin visible propósito doctrinal o tono paródico. (BORGES, 2014: 95)

Tlön è dunque la realizzazione dei mondi possibili che Borges teorizza nel prologo di Ficciones. Ma lo scrittore argentino non si ferma qui; verso la fine del racconto si scopre infatti che Tlön e l'enciclopedia che lo contiene sono opera di una setta misteriosa e in un finale dai toni apocalittici Borges ci fa sapere che a distanza di sette anni dal ritrovamento dell'enciclopedia il mondo reale sta gradualmente prendendo le sembianze di quello di Tlön:

El contacto y el hábito de Tlön han desintegrado este mundo. Encantada por su rigor, la humanidad olvida y torna a olvidar que es un rigor de ajedrecistas, no de ángeles. Ya ha penetrado en las escuelas el (conjetural) «idioma primitivo» de Tlön; ya la enseñanza de su historia armoniosa (y llena de episodios conmovedores) ha obliterado a la que presidió mi niñez; ya en las memorias un pasado ficticio ocupa el sitio de otro, del que nada sabemos con certidumbre - ni siquiera que es falso. (BORGES, 2014: 107)

In questo modo, con un ribaltamento tipicamente borgesiano, l'autore ci mette di fronte al problema del rapporto tra realtà e finzione, tra libro reale e libro fittizio 
e dei confini spesso labili che li dividono. In Tlön, Uqbar, Orbis Tertius Borges ipotizza un mondo in cui la finzione prende gradualmente il posto della realtà, un mondo in cui il mutamento può essere determinato da fattori esterni alla realtà attraverso l'intervento di quella che è la finzione per eccellenza, ossia la narrazione letteraria. Il libro fittizio serve allora a Borges come strumento di riflessione filosofica su un punto che egli sente centrale per la sua scrittura e per il suo pensiero.

\section{Libri fittizi per autori fittizi}

La relazione tra la scrittura di Borges e il libro fittizio non si limita però a questo. Abbiamo già citato - oltre alla funzione fantastica - le sue bibliografie in parte immaginarie, con le quali ritorna da una parte all'invenzione delle fonti cervantina e dall'altra alla bibliografia come gioco, più tipica della linea rabelesiana. Rimane da dire che Borges è l'esponente principale anche di un'altra tendenza ascrivibile all'universo del libro fittizio, che è quella dell'invenzione di libri fittizi che siano funzionali alla caratterizzazione di un personaggio-scrittore anch'esso fittizio. È il caso di Pierre Menard, famoso protagonista di un racconto di Ficciones, spesso ricordato per il suo tentativo di riscrittura del Don Quijote, ma a cui Borges attribuisce anche dei libri fittizi. Tale funzione è poi evidente nel racconto Examen de la obra de Herbert Quain, sempre in Ficciones.

Se Borges è il principale artefice di questa tendenza, tuttavia possiamo individuare un antecedente nel romanzo Max Havelaar (1860) di Multatuli, pseudonimo dello scrittore olandese Eduard Douwes Dekker. Max Havelaar, poeta e scrittore, nonché protagonista del romanzo, manda all'amico Droogstoppel un plico di manoscritti nei quali sono contenute le sue opere. Di esse il romanzo riporta i titoli sotto forma di catalogo, tornando così alla forma dell'elencazione di origine rabelesiana che sarà usata spesso sia da Borges che dagli altri scrittori che si occuperanno di inventare libri fittizi per autori fittizi. La tendenza avrà infatti molta fortuna nel corso del Novecento e bisognerà citare almeno i racconti contenuti in Llamadas telefonicas di Roberto Bolaño e quelli de La sinagoga degli iconoclasti di Juan Rodolfo Wilcock. In questi casi l'espediente del libro fittizio è funzionale alla caratterizzazione dello scrittore fittizio e concorre a delinearne il carattere. Il libro fittizio diventa insomma un espediente centrale per il genere della biografia immaginaria.

In altri casi poi i libri fittizi attributi ad autori fittizi assumono un ruolo più particolare, come in La literatura nazi en América di Bolaño. Qui la funzione di libro fittizio si allarga alla sfera politica, entrando per la prima volta nel terreno della militanza. Con questa raccolta di racconti Bolaño costruisce un immaginario catalogo della letteratura nazista in America Latina, non privo di riferimenti alla realtà. Si tratta quindi di un uso molto pratico del libro fittizio, che entra con forza nella sfera del reale, quella sfera che a un primo approccio gli appartiene in misura minore. 


\section{La funzione metaletteraria: da Calvino a J. J. Abrams}

Se c'è una linea che gioca sull'invenzione degli autori fittizi, Calvino nel suo Se una notte d'inverno un viaggiatore punta invece sulla creazione di lettori fittizi, che vengono a coincidere con i personaggi del Lettore e della Lettrice, e li mette sulle tracce di una serie di libri, anch'essi fittizi, libri che non si trovano e si sottraggono alla lettura, frustrando l'aspettativa dei lettori-personaggi e di quelli reali. I libri fittizi si interrompono dopo poche pagine e il Lettore e la Lettrice cercano disperatamente di trovarne la continuazione; ma ogni pista, ogni possibile collegamento e spunto si rivelano fallaci e dirottano il Lettore e la Lettrice verso un altro libro, diverso da quello precedente, e anzi spesso opposto.

All'interno di questo gioco letterario i confini tra libro reale e libro fittizio ancora una volta si sfaldano. La ricerca del Lettore inizia proprio da Se una notte d'inverno un viaggiatore, che è il titolo sia del libro reale che del primo dei libri fittizi presentati. Siamo di fronte a un gioco a incastro, in cui il lettore viene portato allo scoperto e diventa personaggio e i libri invece si nascondono. C'è un continuo gioco metaletterario, in cui il libro reale si mette in mostra e diventa un libro fittizio, esso stesso oggetto della narrazione e quindi elemento finzionale.

Dei libri fittizi che il Lettore e la Lettrice inseguono appaiono solo gli incipit. La dimensione fittizia di questi inizi di romanzi passa attraverso le tecniche con cui il discorso è riportato. Quelli presentati da Calvino non sono semplicemente libri monchi, che si interrompono, ma sono dei veri e propri libri fittizi perché anche i loro incipit, unica traccia che ne resta, sono raccontati piuttosto che trascritti. C'è sempre, o quasi, un filtro che media tra il libro fittizio, assente, e il racconto che ne viene riportato sulla pagina. Sarà utile allora accennare ad alcune delle tecniche utilizzate da Calvino per creare questo effetto. Per i primi due incipit l'autore utilizza il discorso riportato; c'è un narratore che riporta ciò che il romanzo racconta. Questo appare evidente dall'attacco del primo di essi: «Il romanzo comincia in una stazione ferroviaria» (CALVINO, 1979: 13). Andando avanti nella lettura ci rendiamo conto che chi riporta il racconto non è il narratore o il Lettore, ma i personaggi stessi del libro, protagonisti della storia che viene raccontata. Essi si rivolgono direttamente al Lettore, sottolineando continuamente il perché qualcosa è scritto in un determinato modo e l'atmosfera che l'autore voleva suscitare attraverso la scrittura. Gli esempi di tale procedimento sono moltissimi, ne riportiamo solo alcuni. Alla fine di una lunga descrizione leggiamo: «Tutti questi segni convergono nell'informare che si tratta di una piccola stazione di provincia» (Ibidem). In alcuni casi il personaggio-narratore sembra addirittura insofferente verso i vezzi descrittivi dell'autore e taglia corto: «Il romanzo qui riporta brani di conversazione che sembra non abbiano altra funzione di rappresentare la vita quotidiana d'una città di provincia» (CALVINO, 1979: 19); mentre in altri passi si mette nell'ottica del lettore: «La tua attenzione di lettore ora è tutta rivolta alla donna, è già da qualche pagina che le giri intorno, che io, no, che l'autore gira intorno a questa presenza femminile» (CALVINO, 1979: 21). Il personaggio insomma rivela in maniera quasi spudorata le tecniche descrittive, di immedesimazione e di formazione dell'aspettativa che l'autore del romanzo fittizio avrebbe utilizzato. La funzione di questo tipo di scrittura può essere 
senz'altro definita come metaletteraria; ma tale funzione si esprime anche in altri modi negli incipit dei libri fittizi. Il terzo incipit, Sporgendosi da una costa scoscesa, viene presentato al Lettore attraverso la traduzione orale dal cimmerio fatta in modo estemporaneo del Professor Uzzi-Tuzii, mentre il quarto viene anch'esso letto ad alta voce, ma senza la mediazione della traduzione. Questi esempi mostrano come attraverso i libri fittizi Calvino ragioni sulle modalità di fruizione possibili di un testo, sulle tecniche sottese alla creazione di un prodotto letterario e sul ruolo dell'autore, del lettore e dei personaggi.

Il romanzo di Calvino è insomma un esempio perfetto dell'utilizzo del libro fittizio in funzione metaletteraria, ma questo tipo di funzione era già presente. La ritroviamo ad esempio in Cien años de soledad di Gabriel García Marquez, in cui il misterioso libro che lo zingaro Melquiades scrive per anni si rivela nel finale essere la storia stessa della stirpe Buendía. In questo tipo di letteratura i confini tra reale e fittizio si sfaldano, come già accadeva con Borges. Esempio estremo della caduta della barriera tra libro fittizio e libro reale è il romanzo $S$. La nave di Teseo (2013) scritto da Doug Dorst ma concepito dal regista J. J. Abrams. Qui la simulazione è spinta al suo estremo e il libro fittizio viene a coincidere con il libro reale perfino nella dimensione materiale dell'oggetto libro. L'opera di Abrams si presenta infatti come un libro a più livelli, in cui la pagina è occupata da un corpo centrale, che è quello del libro fittizio, un manoscritto misterioso ritrovato da due studenti, mentre il libro reale, la storia dei due studenti, finisce relegato nei margini sotto forma di commenti scritti da due personaggi negli spazi bianchi del manoscritto. Se l'espediente del manoscritto ritrovato presenta il libro reale come trascrizione, più o meno completa e fedele, di un libro fittizio, nell'opera di Adams il libro fittizio finisce per coincidere pienamente con il libro reale, in una sovrapposizione che annulla il confine tra falsità e verità.

\section{Conclusioni}

I casi qui mostrati non hanno nessuna pretesa di completezza, anzi molti altri se ne sarebbero potuti analizzare e molto più si sarebbe potuto dire su ognuno di essi. Questo breve excursus vuole essere solo una piccola mostra della grande fortuna dall'espediente del libro fittizio nella letteratura moderna e contemporanea. Centrali per comprendere tale longevità sono le funzioni che il libro fittizio ha ricoperto nel corso della sua storia. Se fino al XIX secolo esse sono principalmente quella ludico-satirica e quella legittimante, nel Novecento il libro fittizio accoglie le più diverse sfaccettature: da contenitore per la creazione di mondi fantastici a spunto di riflessione metaletteraria. Altro elemento interessante che emerge, utile per future riflessioni sul tema, è la dialettica che si instaura tra il libro reale e il libro fittizio. Il risultato di questo dialogo sempre in bilico è il dubbio: il lettore in molti casi non riesce a capire se il libro di cui si sta parlando è reale o fittizio; questo determina un effetto straniante e finisce per mettere in relazione il libro fittizio con gli studi sulla letteratura fantastica, in particolare le teorie di Tzvetan Todorov, che basano il fantastico proprio sull'elemento del dubbio e della commistione tra realtà e irrealtà. 
Se questi sono punti da approfondire, aperti per future indagini, quello che invece è evidente è la grande longevità e le numerose modificazioni che il libro fittizio ha saputo affrontare con successo nel corso della sua storia. L'opera di Abrams, attraverso lo sfaldarsi definitivo del confine tra libro reale e libro fittizio, segna l'ideale punto di arrivo di questa lunga storia. L'espediente del libro fittizio implode su sé stesso, come il mondo di Macondo nel momento in cui Aureliano Babilonia legge le ultime pagine del libro di Melquiades in Cien años de soledad. Ma nel caso del libro fittizio come espediente letterario non si tratta di un finale irrevocabile, poiché ancora possibile rimane una sua prosecuzione sotto nuove forme o il suo riutilizzo nei modi di cui qui si è cercato di dare conto. La longevità e il successo del libro fittizio nel corso della storia stanno infatti proprio in questo, nella capacità che gli scrittori hanno avuto di reinventare questo espediente letterario per adattarlo alle necessità e alle sfide poste dalle varie epoche.

\section{BIBLIOGRAFIA}

Albani Paolo (2002), Cataloghi di libri immaginari, in : L'oggetto libro 2001, Edizioni Sylvestre Bonnard, Milano, 2002, pp. 200-215.

Albani Paolo ; Della Bella Paolo (2003), Mirabilia. Catalogo ragionato di libri introvabili, Zanichelli.

BeERBOHM Max (1914), Books within books, in : Id. (1920) And Even Now (Essays), London, William Heinemann, pp. 101-118.

Blumenthal Walter Hart (1966), Imaginary Books and Phantom Libraries, Philadelphia, George S. MacManus Company.

BorgES Jorge Luis (2014), Cuentos completos, Barcellona, Debolsillo.

BRUNET Gustave (1851), Essai sur les bibliothèques imaginaires, Paris, Imprimerie de Ch. Lahure et Cie.

CALVINO Italo (1979), Se una notte d'inverno un viaggiatore, Milano, Mondadori.

CAmmarota Domenico (1986), Gli Pseudobiblia di Chtulhu, in : Gianni Pilo, L'orrore di Chtulhu, Roma, Fanucci, pp. 217-233.

CERVAnTES Miguel de (2005), El ingenioso hidalgo Don Quixote de la Mancha, Madrid, Espasa Calpe.

DE TURRIS Gianfranco, FUSCO Sebastiano (1975), Gli pseudobiblia nella letteratura fantastica, in : Robert William Chambers (ed.), Il re in giallo, Roma, Fanucci, pp. 7-28.

GUADALUPI Gianni (1997), Biblioteche immaginarie, in: DI GIURO Vittorio (ed.), Manuale enciclopedico della bibliofilia,

Fumagalli Giuseppe (1892), Delle biblioteche immaginarie e dei libri che non esistono, Milano, Tipografia Lombardi.

FuMAGALLI Giuseppe Fumagalli, OLSCHKI Leo, Biblioteche immaginarie e roghi di libri.

PALAZZI Roberto (2002), Il labirinto dei libri falsi, inesistenti e immaginari. Alcune storie e qualche esempio, in : MISITI Maria Cristina (ed.), Collezionismo, restauro e antiquariato libraio, Milano, Sylvestre Bonnard, pp. 331-358. 
SERRAI Alfredo (1993), Cataloghi fantastici, in : Storia della bibliografia, vol. IV, Roma, Bulzoni, pp. 272-280.

SPRAGUE DE CAMP Lyon (1947), The unwritten classics, in : «The Saturday Review of Literature», 29 ${ }^{\text {th }}$ March 1947.

TUZZI Hans (1997), Cataloghi immaginari, in: DI GIURO Vittorio (ed.), Manuale enciclopedico della bibliofilia,

WEBSTER SPARGO John (1952), Imaginary books and libraries, an essay in lighter vein, Chicago, Caxton Club. 